\title{
Droplet-Free Digital Enzyme-Linked Immunosorbent Assay Based on a Tyramide Signal Amplification System
}

Kenji Akama*, Kentaro Shirai, Seigo Suzuki

Sysmex Corporation, 4-4-4 Takatsukadai, Nishi-ku, Kobe 651-2271, Japan.

* E-mail: Akama.Kenji @ sysmex.co.jp

Fax: 81-78-992-3284

\section{Table of Contents}

Supplementary Figure 1. Typical overlay image of fluorescence and bright field images of beads with captured HBsAg at $\lambda$ equal to 0.1 using a HRP-tyramide reaction............................................ -2

Supplementary Figure 2. Time course of the average number of tyramide molecules deposited on each

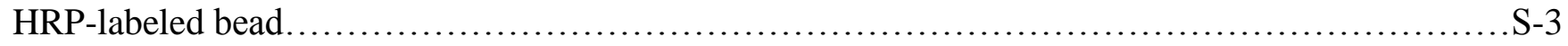

Supplementary Figure 3. Typical plot of SSC and fluorescence intensity of all detected beads at differ-

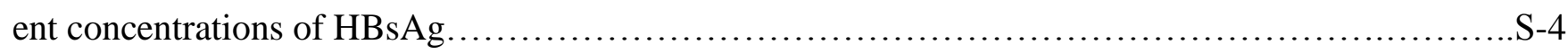

Supplementary Figure 4. HBsAg detection by droplet-based Digital ELISA using the HRP-Quantared

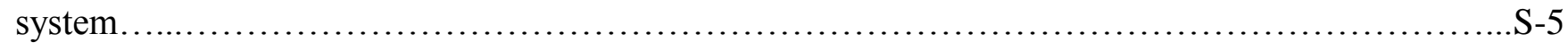

Supplementary Figure 5. Calibration curve for HBsAg by droplet-based Digital ELISA using the HRP-Quantared system. S-6 


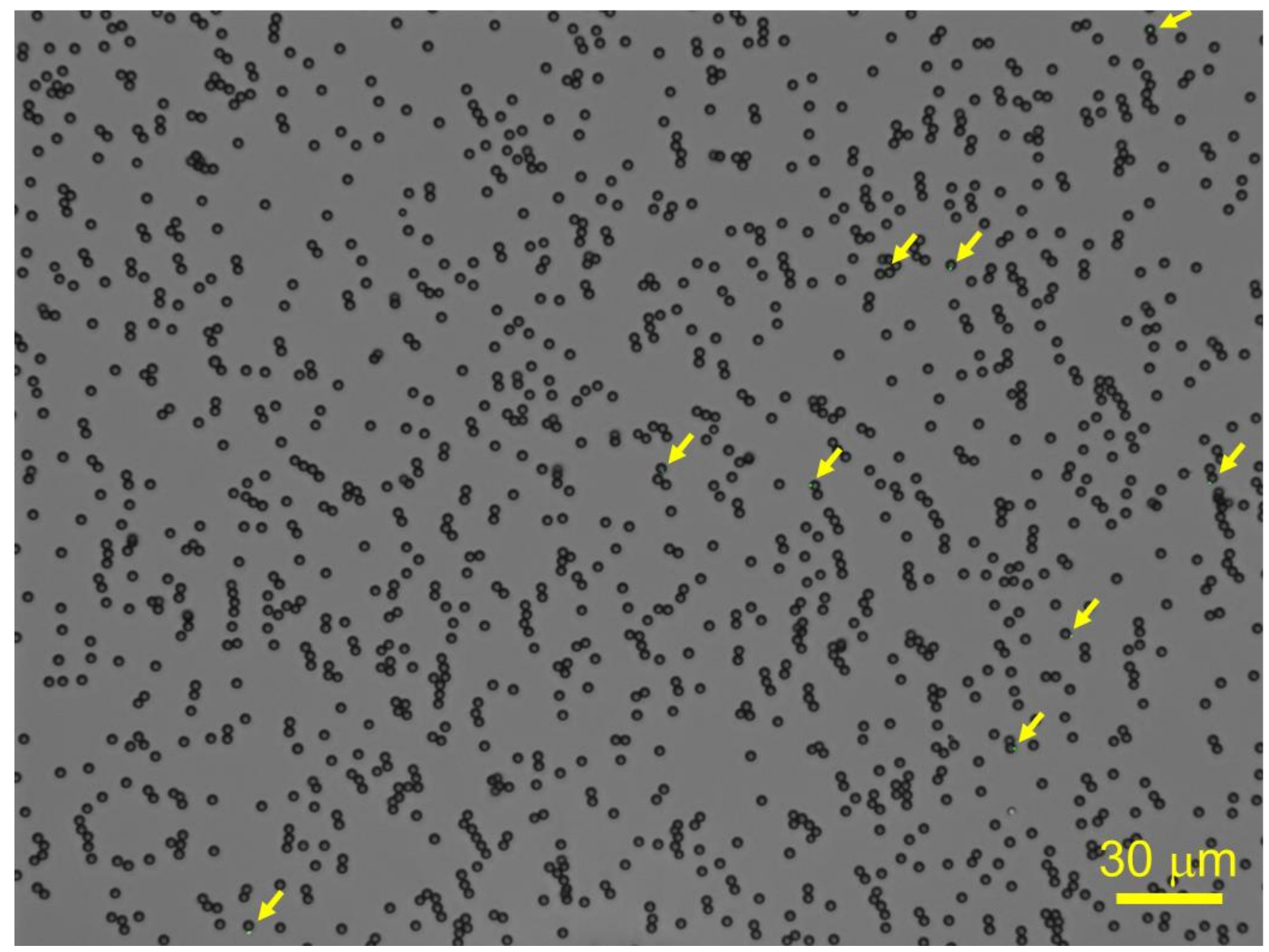

Supplementary Figure 1. Typical overlay image of a fluorescence image and bright field image of beads with captured HBsAg at $\lambda$ equal to 0.1 using a HRP-tyramide reaction. Yellow arrows indicate bright spots. 


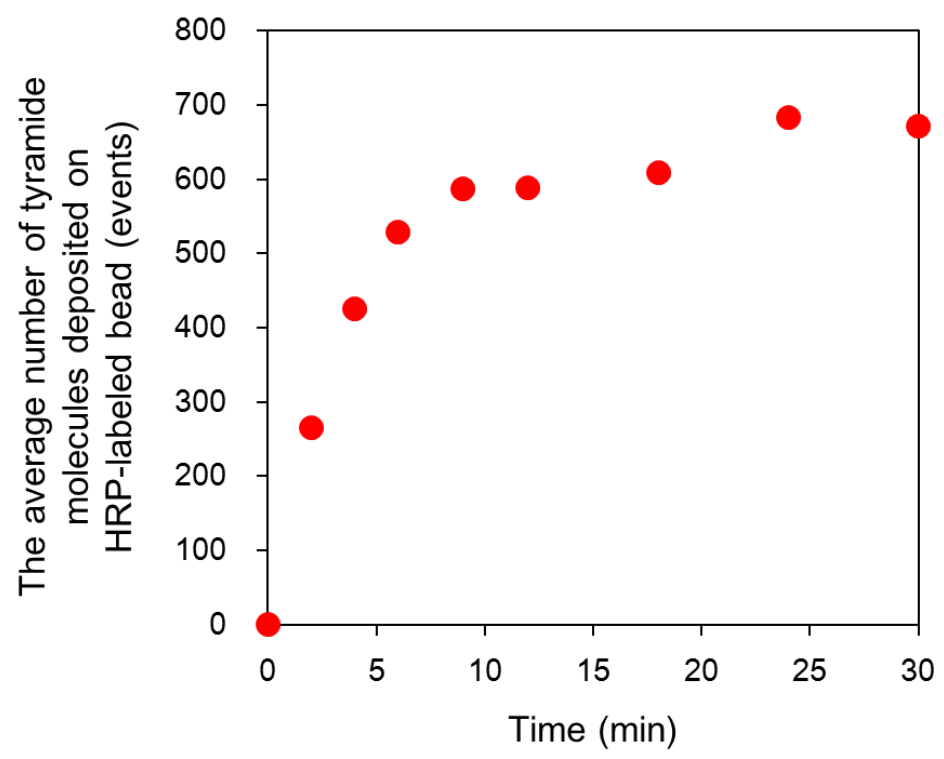

Supplementary Figure 2. Time course of the average number of tyramide molecules deposited on each HRP-labeled bead. After the reaction of HRP with tyramide, the amount of tyramide in the supernatant was quantified and the amount of tyramide deposited on each bead was calculated. 
a)

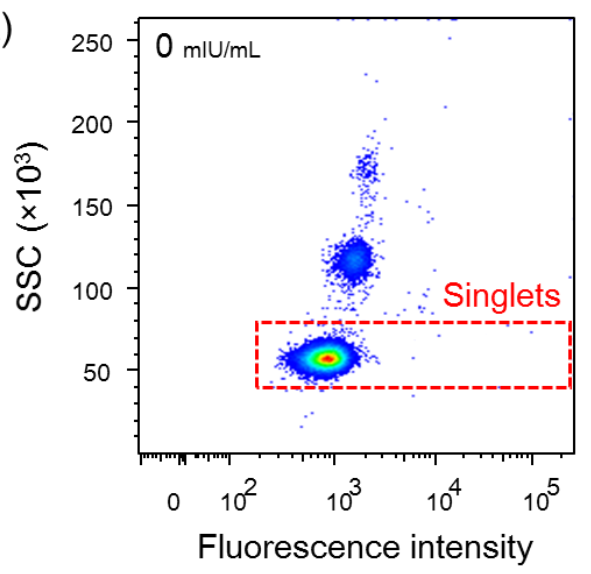

c)

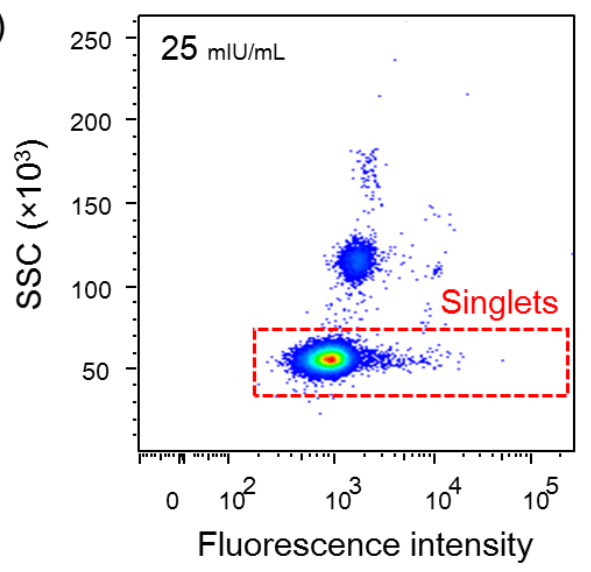

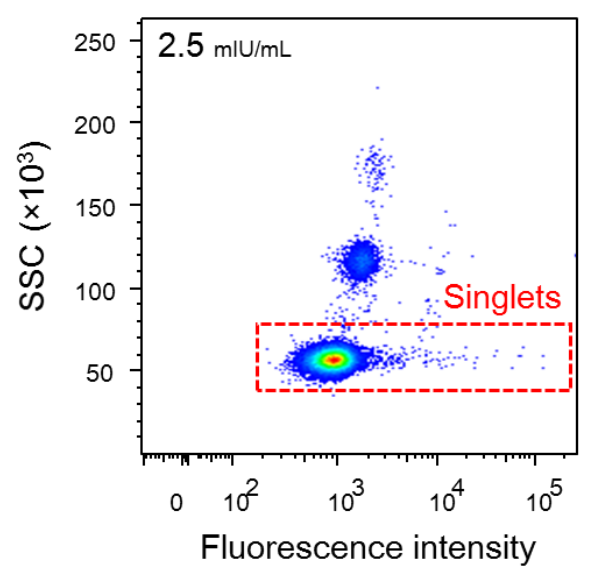

d)

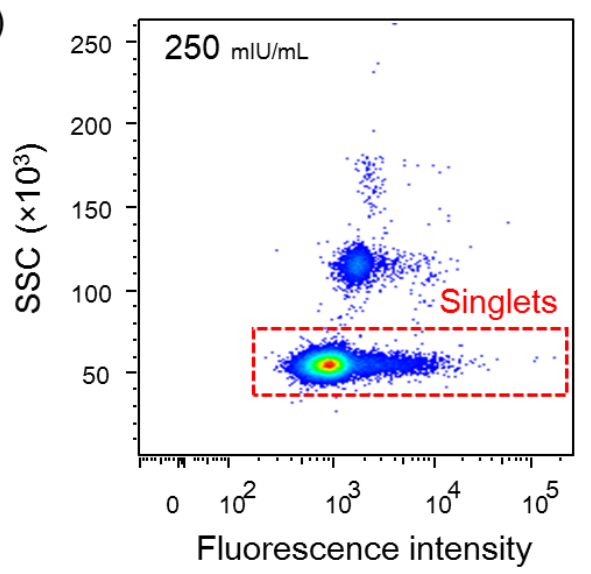

Supplementary Figure 3. Typical plots of SSC and fluorescence intensity for all detected beads at different concentrations of HBsAg (excitation: $404 \mathrm{~nm}$; emission: 425-470 nm). 

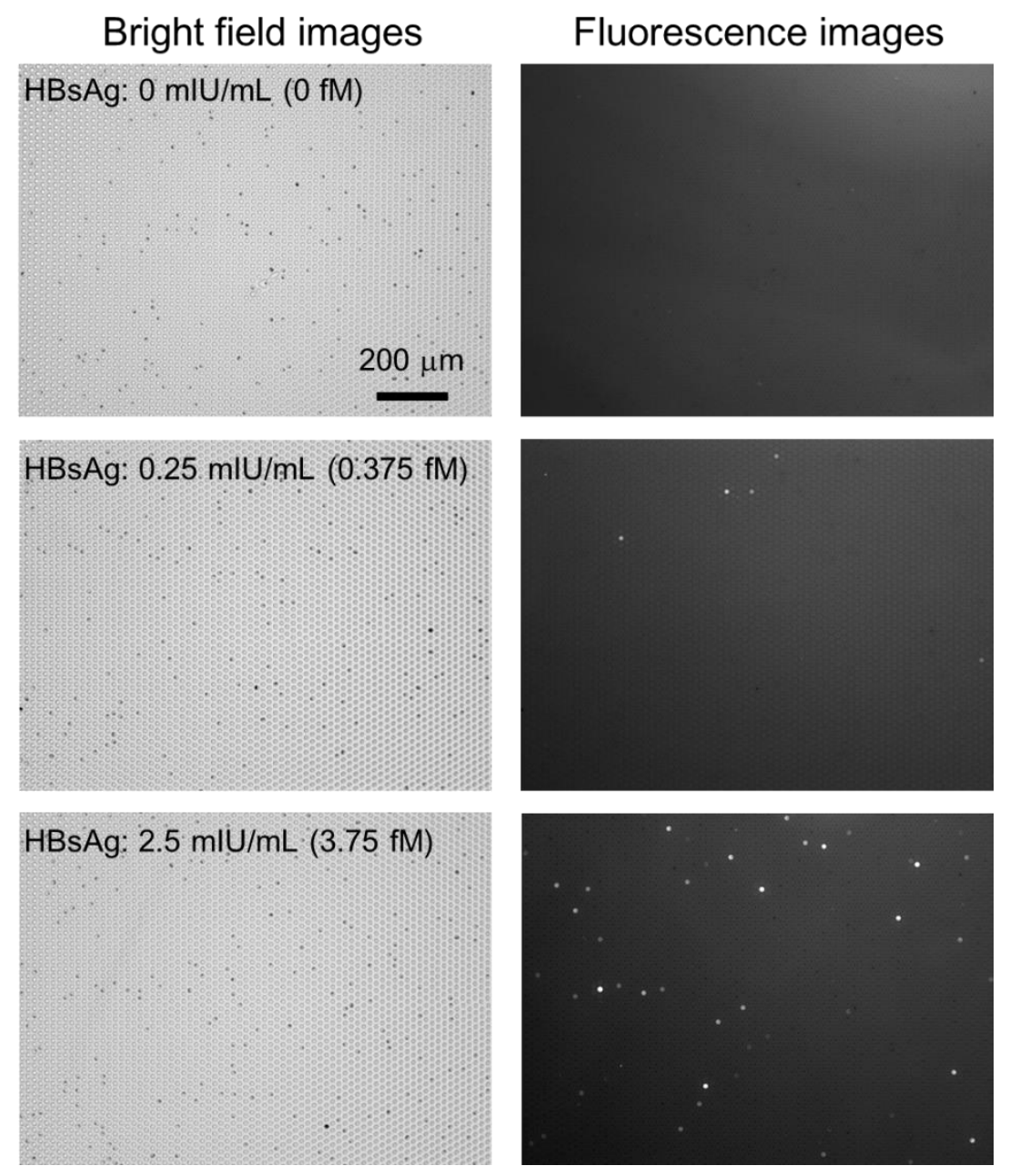

Supplementary Figure 4. HBsAg detection by droplet-based Digital ELISA using the HRP-Quantared system. The beads were loaded into a femtoliter droplet array together with Qantared, then 40 fluorescence images and bright field images of different fields of view (FOV) were taken for each sample. The left column shows portions of typical bright field images and the right column shows portions of typical fluorescence images (excitation: 520-580 nm; emission: 593-668 nm). The average number of beads in each FOV was over 2,000 and the total number of beads analyzed was over 80,000. 


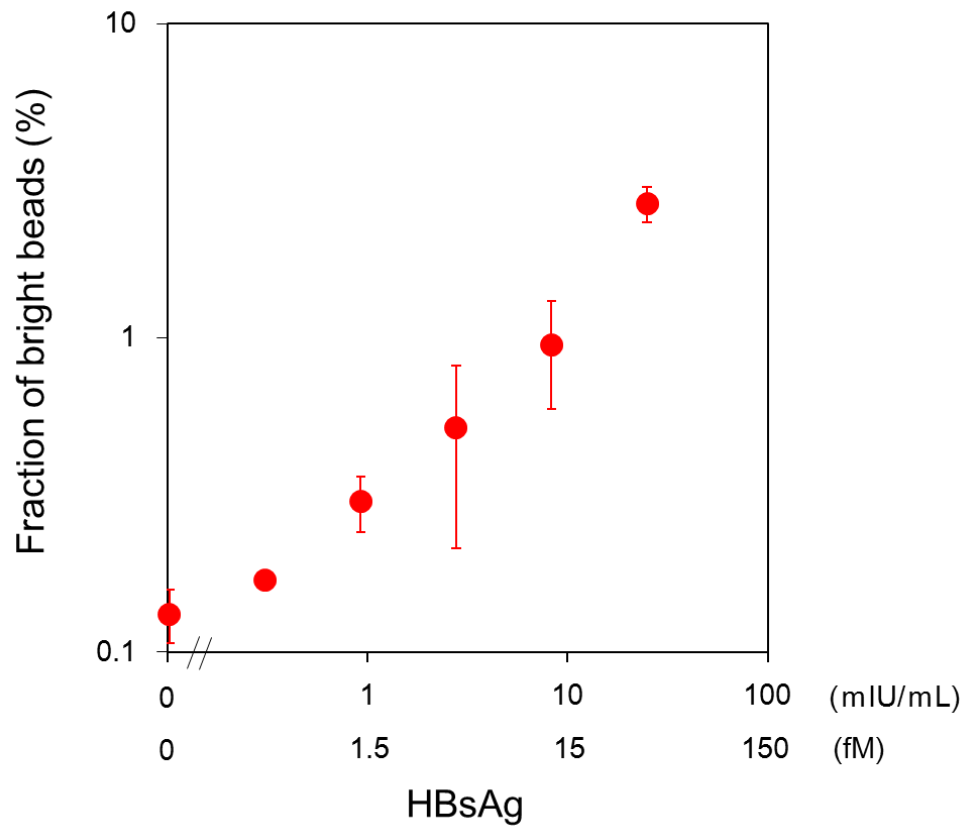

Supplementary Figure 5. Calibration curve for HBsAg by droplet-based Digital ELISA using the HRP-Quantared system. $\mathrm{N}=4$ and error bars represent 2 times the SD. 